\title{
ON THE STONE-WEIERSTRASS THEOREM FOR THE STRICT AND SUPERSTRICT TOPOLOGIES
}

\author{
R. G. HAYDON
}

\begin{abstract}
Sentilles has introduced topologies $\beta_{0}, \beta$ and $\beta_{1}$ on the space $C_{b}(S)$ of all bounded, continuous, real-valued functions on the completely regular space $S$, which yield as dual spaces the three important spaces of measures, $M_{t}(S), M_{\tau}(S)$ and $M_{\sigma}(S)$, respectively. A number of authors have proved a Stone-Weierstrass theorem for $\beta_{0}$, the coarsest of the three topologies. In this paper, it is shown that the superstrict topology $\beta_{1}$ does not obey the Stone-Weierstrass theorem, except perhaps when $\beta_{1}=\beta$. Examples are then given to show that the situation for $\beta$ itself is rather complicated.
\end{abstract}

1. Introduction. The topological spaces $S, T, \ldots$ considered in what follows will all be completely regular (and Hausdorff). The space $C_{b}(S)$ of all bounded, continuous, real-valued functions on $S$ may be equipped with any one of the strict, superstrict and substrict topologies, denoted by $\beta, \beta_{1}$ and $\beta_{0}$, respectively. These were introduced by Sentilles in [7], and by Fremlin, Garling and Haydon in [2], where the notations $T_{\tau}, T_{\sigma}$ and $T_{t}$ were used. A convenient definition is that of [2], in terms of uniformly smooth sets of functionals. If $\left(f_{\alpha}\right)$ is a decreasing net in $C_{b}(X)$, with pointwise infimum 0 , let us write $f_{\alpha} \downarrow 0$. Then a set $A$ of linear functionals on $C_{b}(X)$ is uniformly $\tau$ smooth (resp. uniformly $\sigma$-smooth) if $\left\langle\mu, f_{\alpha}\right\rangle \rightarrow 0$ (resp. $\left\langle\mu, f_{n}\right\rangle \rightarrow 0$ ) uniformly over $\mu \in A$, whenever $f_{\alpha} \downarrow 0$ (resp. whenever $\left(f_{n}\right)$ is a sequence in $C_{b}(X)$ and $f_{n} \downarrow 0$ ). The topology $\beta$ (resp. $\beta_{1}$ ) is defined to be the topology of uniform convergence on uniformly $\tau$-smooth (resp. uniformly $\sigma$-smooth) sets of functionals. The dual of $C_{b}(S)$ under $\beta$ (resp. $\beta_{1}$ ) can be identified with the space $M_{\tau}(S)$ (resp. $M_{\sigma}(S)$ ) of all $\tau$-additive Borel measures on $S$ (resp. all $\sigma$-additive Baire measures on $S$ ) (cf., for instance, $\$ 2$ of [4]). When we make this identification, it is a useful convention to write $\langle\mu, f\rangle$ when $f \in C_{b}(S)$ and we are thinking of $\mu$ as a linear functional, and to write $\mu(F)$ when $F$ is a $\mu$ measurable set and we are thinking of $\mu$ as a set function.

The topology $\beta_{0}$ can be defined as the finest locally convex topology on $C_{b}(S)$ that coincides on the (uniform norm) unit ball with the topology $K$ of uniform convergence on compact subsets of $S$. This topology is coarser than $\beta$, and the dual $\left(C_{b}(S), \beta_{0}\right)^{\prime}$ can be identified with the space $M_{t}(S)$ of all tight measures on $S$. The $\beta_{0}$-equicontinuous sets are the uniformly tight sets of measures, that is, those norm bounded sets $A$ having the property: for every $\varepsilon>0$, there exists a compact set $K \subseteq S$ such that $|\mu|(S \backslash K)<\varepsilon$ for all $\mu \in A$.

Received by the editors October 3, 1974.

AMS (MOS) subject classifications (1970). Primary 46E10; Secondary 28A30. 
The dual of $C_{b}(S)$ under the uniform norm topology can, of course, be identified with the space $M(\beta S)$ of all Radon measures on the Stone-Čech compactification $\beta S$ on $S$. Let us write $\mu^{\beta}$ for the measure on $\beta S$ associated with $\mu \in\left(C_{b}(S),\|\cdot\|\right)^{\prime}$. Then, by the results of $\$ 2$ of [4],

$\mu$ is $\tau$-smooth iff $\mu^{\beta}(K)=0$ whenever $K$ is a compact subset of $\beta S \backslash S$;

$\mu$ is $\sigma$-smooth iff $\mu^{\beta}(K)=0$ whenever $K$ is a compact $G_{\delta}$ subset of $\beta S$ and $S \cap K=\varnothing$;

$\mu$ is tight iff $S$ is $\mu^{\beta}$-measurable with $\left|\mu^{\beta}\right|(\beta S \backslash S)=0$.

If $f \in C_{b}(S)$, we denote by $f^{\beta}$ the unique continuous extension of $f$ to $\beta S$.

Of the three principal classes of measures, the tight measures are certainly the easiest to handle in practice, since we have available the powerful method of reduction to the case of measures on a compact space. But it has been suggested by more than one author (e.g. [4], [10], [12]) that the $\tau$-additive measures are "more natural". It is reasonable, therefore, to see how many of the agreeable properties of tight measures, and of the associated topology $\beta_{0}$, extend to $\tau$-smooth measures and the strict topology $\beta$. The starting point in this paper is the Stone-Weierstrass theorem for $\beta_{0}$, which can be found in [2], [3], [8] and [11]. Summers has used this theorem in his investigation of separability [9], and has drawn attention to the question of whether such a theorem holds for $\beta$.

We shall be able to see quickly that $\beta_{1}$ will not satisfy a theorem of this type, except perhaps when $\beta_{1}=\beta$. We shall note an example of a separable metric space $S$ such that $\left(C_{b}(S), \beta\right)$ does not satisfy the Stone-Weierstrass theorem, and then construct a separable metric space $T$ such that $M_{\tau}(T) \neq M_{t}(T)$, while $\left(C_{b}(T), \beta\right)$ does satisfy the theorem. The unsatisfactory nature of these results for $\beta$ must, in the author's opinion, be taken as evidence against the claim that the class of $\tau$-smooth measures should be our principal object of study.

2. The Stone-Weierstrass property. Let us say that a locally convex topology $\gamma$ on $C_{b}(S)$ has the property $(\mathrm{SW})$ if, whenever $A$ is a subalgebra of $C_{b}(S)$, which separates the points of $S$ and contains the constant functions, it follows that $A$ is $\gamma$-dense in $C_{b}(S)$. This is a rather weaker property than is generally considered. Let us say, then, that $\gamma$ has the slightly stronger property (SSSW) if $A$ is $\gamma$-dense in $C_{b}(S)$ whenever $A$ is a separating subalgebra such that, for each $s \in S$, there is some $f \in A$ with $f(s) \neq 0$. Each of these is, of course, more properly a property of the dual $\left(C_{b}(S), \gamma\right)^{\prime}$, since any two locally convex topologies that yield the same dual have the same closed subspaces. The first proposition of this paragraph shows that the properties (SW) and (SSSW) are equivalent for a fairly wide class of topologies. For convenience, let us say that a locally convex topology $\gamma$ on $C_{b}(S)$ is admissible if $\gamma$ is coarser than the uniform norm topology and finer than the topology $\tau_{p}$ of pointwise convergence on $S$, and if $\left(C_{b}(S), \gamma\right)^{\prime}$ is a solid subspace of $M(\beta S)$ (that is to say, if $\mu \in\left(C_{b}(S), \gamma\right)^{\prime}, \nu \in M(\beta S)$ and $|\nu| \leq|\mu|$ imply $\left.\nu \in\left(C_{b}(S), \gamma\right)^{\prime}\right)$. This will be the case if $\gamma$ is a strict topology, or if $\gamma$ is any locally convex topology, finer than $\tau_{p}$, for which multiplication is separately continuous, and such that $\left(C_{b}(S), \gamma\right)^{\prime}$ is a norm-closed subspace of $M(\beta S)$.

2.1 THEOREM. The properties (SW) and (SSSW) are equivalent for admissible 
topologies. Moreover, if $\gamma$ is admissible and $\left(C_{b}(S), \gamma\right)$ has $(\mathrm{SW})$, then $\left(C_{b}(S), \gamma\right)^{\prime}$ $\subseteq M_{\tau}(S)$.

Proof. Let us prove the last assertion first. If $\left(C_{b}(S), \gamma\right)^{\prime} \nsubseteq M_{\tau}(S)$, there exist $\mu \in\left(C_{b}(S), \gamma\right)^{\prime}$ and a compact $K \subseteq \beta S \backslash S$ with $\mu^{\beta}(K) \neq 0$. Choose any point $s_{0} \in S$ and define $A=\left\{f \in C_{b}(S): f^{\beta} \mid K=f\left(s_{0}\right)\right\}$. Then $A$ is a separating subalgebra and $1_{S} \in A$. Since $\left(C_{b}(S), \gamma\right)^{\prime}$ is solid, the measure $\nu$, defined by $\nu^{\beta}=1_{K_{1}} \cdot \mu^{\beta}$, is in $\left(C_{b}(S), \gamma\right)^{\prime}$. Also $\delta\left(s_{0}\right) \in\left(C_{b}(S), \gamma\right)^{\prime}$ and $\lambda$ $=\delta\left(s_{0}\right)-\left(\mu^{\beta}(K)\right)^{-1} \nu$ is a nonzero element of $\left(C_{b}(S), \gamma\right)^{\prime}$ with $\lambda \mid A=0$.

Suppose now that $\left(C_{b}(S), \gamma\right)$ does not satisfy (SSSW). Then there exists a subalgebra $A$ of $C_{b}(S)$, which separates points of $S$ from each other and from 0 , together with a nonzero element $\mu$ of $\left(C_{b}(S), \gamma\right)^{\prime}$ with $\mu \mid A=0$. Since, by what we have just proved, we know that $\left(C_{b}(S), \gamma\right)$ does not satisfy (SW) if $\left(C_{b}(S), \gamma\right)^{\prime} \nsubseteq M_{\tau}(S)$, we can suppose that $\mu$ is $\tau$-smooth. So there is a subset $T=\operatorname{supp} \mu=S \backslash \cup\{G: G$ is open in $S$ and $|\mu|(G)=0\}$, with the property $|\mu|(S \backslash T)=0,|\mu|(G)>0$ whenever $G$ is open and $G \cap T \neq \varnothing$. Since $A$ does not annihilate any point of $S, T$ contains more than one point. So there is a function $h \in A$ that is not constant on $T$. In this case, $\mu$ and $\mu_{1}=h \cdot \mu$ are linearly independent elements of $\left(C_{b}(S), \gamma\right)^{\prime}$. Putting

$$
B=\left\{f+\alpha 1_{S}: f \in A \text { and } \alpha \in \mathbf{R}\right\},
$$

we see that $B$ is a separating subalgebra of $C_{b}(S)$ and that $1_{S} \in B$, while, for suitably chosen $\alpha, \alpha_{1} \in \mathbf{R}, \lambda=\alpha \mu+\alpha_{1} \mu_{1}$ is a nonzero element of $\left(C_{b}(S), \gamma\right)^{\prime}$ with $\lambda \mid B=0$.

2.2 Corollary. If $\left(C_{b}(S), \beta_{1}\right)$ has the property (SW), then necessarily $\left(C_{b}(S), \beta_{1}\right)=\left(C_{b}(S), \beta\right)$.

Proof. We know from 2.1 that we must have $M_{\sigma}(S)=M_{\tau}(S)$. The rest follows from Proposition 5 of [2].

The following proposition characterizes (SW) in a useful alternative form. We use the following piece of notation: when $S$ and $T$ are completely regular and $\phi: S \rightarrow T$ is continuous, we define a mapping $\tilde{\phi}: M(\beta S) \rightarrow M(\beta T)$ by

$$
\langle\tilde{\phi} \mu, g\rangle=\langle\mu, g \circ \phi\rangle \quad\left(g \in C_{b}(T)\right)
$$

(identifying, as usual, $M(\beta S)$ with $\left.\left(C_{b}(S),\|\cdot\|\right)^{\prime}\right)$; then $\phi$ takes $M_{\sigma}(S)$ into $M_{\sigma}(T)$, $M_{\tau}(S)$ into $M_{\tau}(T)$ and $M_{t}(S)$ into $M_{t}(T)$. Moreover, $\tilde{\phi} \mu$, as defined above, coincides in these cases with the image measure defined in the usual settheoretic way by $(\tilde{\phi} \mu)(E)=\mu\left(\phi^{-1} E\right)$.

2.3 Proposition. Let $S$ be a completely regular space and let $\gamma$ be a locally convex topology on $C_{b}(S)$, coarser than the uniform norm topology. Then $\left(C_{b}(S), \gamma\right)$ satisfies $(\mathrm{SW})$ if and only if, whenever $T$ is a completely regular space, and $\phi: S \rightarrow T$ is a continuous injection, the map $\tilde{\phi}$ is injective on $\left(C_{b}(S), \gamma\right)^{\prime}$.

Proof. If $\left(C_{b}(S), \gamma\right)$ satisfies (SW) and $\phi$ is as in the statement of the proposition, let us consider $A=\left\{f \circ \phi: f \in C_{b}(T)\right\}$, a separating subalgebra of $C_{b}(S)$, which contains $1_{S}$. If $\tilde{\phi}$ were not injective on $\left(C_{b}(S), \gamma\right)^{\prime}$, there would be some nonzero $\mu \in\left(C_{b}(S), \gamma\right)^{\prime}$ such that $\langle\mu, f \circ \phi\rangle=\langle\tilde{\phi} \mu, f\rangle=0$ for all $f \in C_{b}(T)$.

Conversely suppose that $A$ is a separating subalgebra of $C_{b}(S)$ and that $1_{S} \in A$. Let $B$ denote the norm closure of $A$ and consider the associated 
subalgebra $B^{\beta}=\left\{f^{\beta}: f \in A\right\}$ of $C(\beta S)$. By one of the elementary consequences of the standard Stone-Weierstrass theorem, $B^{\beta}$ has the form $\{g \circ \psi: g \in C(T)\}$ for some compact Hausdorff space $T$ and some continuous surjection $\psi: \beta S \rightarrow T$. Let us define $\phi=\psi \mid S$. Then $\phi$ is an injection, since $A$ separates points. If $A$ were not $\gamma$-dense in $C_{b}(S)$ then there would exist a nonzero $\mu \in\left(C_{b}(S), \gamma\right)^{\prime}$ with $\mu \mid A=0$. Since $\gamma$ is coarser than the norm topology, we should have $\mu \mid B=0$, and thus $\tilde{\phi} \mu=0$.

\subsection{COROLlaRY. The substrict topology $\beta_{0}$ always satisfies (SW).}

Proof. This now follows from the well-known result that if $\phi: S \rightarrow T$ is a continuous injection, then so is $\tilde{\phi} \mid M_{t}(S)$. An easy way to show this is to observe that, if $\mu \in M_{t}(S)$, then $\mu$ is determined by the values $\mu(K)$ with $K$ compact. If $\phi$ is an injection, then $\mu(K)=\mu\left(\phi^{-1} \phi K\right)=(\tilde{\phi} \mu)(\phi K)$.

2.5 REMARK. Even if $\phi$ is a homeomorphic embedding, $\tilde{\phi}$ does not have to be injective on $M_{\sigma}(S)$. Indeed, an example of this phenomenon was, in effect, produced in the proof of the first part of Proposition 2.1. On the other hand, $\tilde{\phi} \mid M_{\tau}(S)$ will always be injective when $\phi$ is a homeomorphic embedding. One way to see this is to recall the correspondence between $\tau$-smooth functionals and Borel (as opposed to Baire) measures. The Borel $\sigma$-field $\mathscr{B}(S)$ of $S$ is exactly $\left\{\phi^{-1} B: B \in \mathscr{B}(T)\right\}$. So if $\tilde{\phi} \mu=0$, then, for every $E=\phi^{-1} B \in \mathscr{B}(S)$, we have $\mu E=\mu\left(\phi^{-1} B\right)=(\tilde{\phi} \mu)(B)=0$.

We are now in a position to give examples showing that the situation for the strict topology $\beta$ is far from straightforward.

2.6 EXAMPLE. A separable metric space $S$ such that $\left(C_{b}(S), \beta\right)$ does not satisfy (SW).

Let $J$ be a subset of $\mathbf{I}=[0,1]$ with $m^{*} J=1$ and $m_{*} J=0, m$ denoting Lebesgue measure. Define

$$
S=(J \times\{0\}) \cup((\mathbf{I} \backslash J) \times\{1\}) \subset \mathbf{I} \times\{0,1\},
$$

and let $\pi: S \rightarrow$ I be the projection on the first coordinate. Lebesgue measure $m$ on $\mathbf{I}$ induces $\tau$-smooth measures $\mu_{0}$ on $J \times\{0\}$ and $\mu_{1}$ on $(\mathbf{I} \backslash J) \times\{1\}$, and we can see that $\mu_{1} \neq \mu_{0}$ while $\tilde{\pi} \mu_{1}=\tilde{\pi} \mu_{0}=m$.

This example is evidently a very special case, in which the space $S$ splits into two subsets which "fit together" nicely. In order to give an example where this phenomenon will not occur, we need the following lemma which constructs a particular nonmeasurable subset of I by a variant on the familiar method of "one for me and one for you". For facts about analytic spaces see [5] and [1].

2.7 LEMmA. There is a nonmeasurable subset $T$ of $[0,1]$ with the following property:

(*) Let $H, K$ be analytic subsets of $[0,1]$, let $Z$ be a uncountable separable metric space and let $\phi: H \rightarrow Z, \psi: K \rightarrow Z$ be continuous surjections. Then there are points $x \in H \cap T, y \in K \cap T$ with $\phi x=\psi y$.

Proof. Let $\delta$ denote the set of all quadruples $(H, K, \phi, \psi)$ such that $H, K$ are analytic subsets of $[0,1]$, while $\phi$ and $\psi$ are continuous mappings from $H$ and $K$, respectively, onto some uncountable subset $Z$ of the product space $I^{\omega}$. Then 
card $\delta=$ card $\mathbf{R}=\mathbf{c}$, and (identifying $\mathbf{c}$ with the corresponding initial ordinal) we can index $\delta$ as $\left\{\left(H_{\alpha}, K_{\alpha}, \phi_{\alpha}, \psi_{\alpha}\right): \alpha<\mathbf{c}\right\}$. We now define families $\left(x_{\alpha}\right)$, $\left(y_{\alpha}\right),\left(z_{\alpha}\right)$ of points of I by transfinite induction.

If $\beta<\mathrm{c}$ and $x_{\alpha}, y_{\alpha}, z_{\alpha}$ have already been defined for $\alpha<\beta$, we first note that card $\phi_{\beta} H_{\beta}=$ card $H_{\beta}=$ card $K_{\beta}=\mathrm{c}$ (since, by 32.V, Corollary 1 of [5], an uncountable analytic set necessarily has cardinality c). So we can choose $x_{\beta} \in H_{\beta}, y_{\beta} \in K_{\beta}$ with $\phi_{\beta}\left(x_{\beta}\right)=\psi_{\beta}\left(y_{\beta}\right)$ and $x_{\beta} \neq z_{\alpha} \neq y_{\beta}(\alpha<\beta)$; we also choose $z_{\beta} \in H_{\beta}$ with $x_{\alpha} \neq z_{\beta} \neq y_{\alpha}(\alpha \leq \beta)$.

Finally, we put

$$
T=\left\{x_{\alpha}: \alpha<\mathbf{c}\right\} \cup\left\{y_{\alpha}: \alpha<\mathbf{c}\right\} .
$$

Whenever $H$ is an uncountable Borel subset of $\mathbf{I}$, we have $H=H_{\alpha}$ for some $\alpha$, and so $x_{\alpha} \in T \cap H$ and $z_{\alpha} \in H \backslash T$. So $H \cap T$ and $H \backslash T$ are both nonempty, and it is thus true that $m^{*} T=1, m_{*} T=0$. But, by construction, $T$ also has the property (*), since every separable metrizable space $Z$ can be homeomorphically embedded in $[0,1]^{\omega}$.

2.8 EXAMPLE. $A$ separable metric space $T$ such that $M_{t}(T) \neq M_{\tau}(T)$ while $\left(C_{b}(T), \beta\right)$ does satisfy $(\mathrm{SW})$.

We take $T$ to be the subset of I constructed in Lemma 2.7. Suppose $X$ is completely regular and let $\eta: T \rightarrow X$ be a continuous injection. Let us put $X_{1}=\eta T$. The map $\tilde{j}: M_{\tau}\left(X_{1}\right) \rightarrow M_{\tau}(X)$ induced by the inclusion $j: X_{1} \rightarrow X$ is injective by the remark of 2.5 , so it will be enough to show that the map $M_{\tau}(T) \rightarrow M_{\tau}\left(X_{1}\right)$ is injective.

Now $X_{1}$ is a regular continuous image of a separable metric space (a cosmic space in the terminology of [6]), so that there is a coarser metrizable topology on $X_{1}$. Let $Y$ denote the set $X_{1}$ equipped with this topology, and let $\hat{Y}$ be the completion of $Y$ with respect to some compatible metric. Write $\theta$ for the continuous map $T \rightarrow \hat{Y}$ induced by $\eta$. By Exercise 8.4 of [1], there is a $G_{\delta}$ subset $\hat{T}$ of I, containing $T$, and a continuous map $\hat{\theta}: \hat{T} \rightarrow \hat{Y}$ extending $\theta$.

If $\tilde{\eta}$ is not injective, then neither is $\tilde{\theta}$ and it is not hard to see that we can find $\mu, \nu$ in $M_{\tau}^{+}(T)$ with $\|\mu\|=\|\nu\|=1, \mu \wedge \nu=0$ and $\tilde{\theta} \mu=\tilde{\theta} \nu=\lambda$, say. Let us write $\hat{\mu}, \hat{\nu}$ for the measures on $\hat{T}$ associated with $\mu, \nu$, respectively. Since $\mu \wedge \nu=0$, we have $\hat{\mu} \wedge \hat{\nu}=0$, so there are disjoint Borel subsets $H_{0}, K_{0}$ of $\hat{T}$ with $\hat{\mu}\left(H_{0}\right)=1, \hat{\nu}\left(K_{0}\right)=1$. The sets $\hat{\theta} H_{0}$ and $\hat{\theta} K_{0}$ are analytic, and thus $\lambda$ measurable. We certainly have $\lambda\left(\hat{\theta} H_{0}\right)=\lambda\left(\hat{\theta} K_{0}\right)=1$, so, putting $Z=\hat{\theta} H_{0}$ $\cap \hat{\theta} K_{0}$, we have $\lambda(Z)=1$. Thus, if $H=H_{0} \cap \hat{\theta}^{-1} Z$ and $K=K_{0} \cap \hat{\theta}^{-1} Z$, $H$ and $K$ are analytic subsets of $I$ with $\hat{\mu}(H)=\hat{\nu}(K)=1$. It follows that $\mu(H \cap T)=\nu(K \cap T)=1$, and, since $\mu$ and $\nu$ cannot both be tight measures (by the (SW) property for $\beta_{0}$ ), at least one of $H \cap T$ and $K \cap T$ is uncountable. Thus $Z$ is uncountable, because $\hat{\theta} \mid T=\theta$ is an injection. We can now apply $(*)$ with $\phi=\hat{\theta} \mid H$ and $\psi=\hat{\theta} \mid K$. The conclusion that there exist $x \in T \cap H$ and $y \in T \cap K$ with $\phi x=\psi y$ contradicts the hypothesis that $\theta$ is an injection and concludes the proof.

\section{REFERENCES}

1. G. Choquet, Lectures on analysis. Vol. I: Integration and topological vector spaces, Benjamin, New York, 1969. MR 40 \#3252; erratum, 44, p. 1630. 
2. D. H. Fremlin, D. J. H. Garling and R. G. Haydon, Bounded measures on topological spaces, Proc. London Math. Soc. (3) 25 (1972), 115-136.

3. R. Giles, A generalization of the strict topology, Trans. Amer. Math. Soc. 161 (1971), 467474. MR 43 \#7919.

4. J. D. Knowles, Measures on topological spaces, Proc. London Math. Soc. (3) 17 (1967), 139156. MR 34 \#4441.

5. C. Kuratowski, Topologie. Vol. I, 2nd ed., PWN, Warsaw, 1948; English transl., PWN, Warsaw; Academic Press, New York, 1966. MR 10, 389; 40 \# 840.

6. E. A. Michael, $\aleph_{0}$-spaces, J. Math. Mech. 15 (1966), 983-1002. MR 34 \#6723.

7. F. D. Sentilles, Bounded continuous functions on a completely regular space, Trans. Amer. Math. Soc. 168 (1972), 311-336. MR 45 \#4133.

8. W. H. Summers, The general complex bounded case of the strict weighted approximation problem, Math. Ann. 192 (1971), 90-98. MR 44 \#2024.

9. - Separability in the strict and substrict topologies, Proc. Amer. Math. Soc. 35 (1972), 507-514. MR 46 \#2410.

10. F. Topsøe, Topology and measure, Springer-Verlag, Berlin, 1970.

11. A. C. M. van Rooij, Tight functionals and the strict topology, Kyungpook Math. J. 7 (1967), 41-43. MR 37 \#3336.

12. V. S. Varadarajan, Measures on topological spaces, Mat. Sb. 55 (97) (1961), 35-100; English transl., Amer. Math. Soc. Transl. (2) 48 (1965), 161-228. MR 26 \#6342.

Mathematical Institute, 24-29 St. Giles, Oxford, OX1 3LB, England 